ARTICLE

\title{
Identification of a plastidial phenylalanine exporter that influences flux distribution through the phenylalanine biosynthetic network
}

Joshua R. Widhalm', Michael Gutensohn², Heejin Yoo1,2, Funmilayo Adebesin, Yichun Qian,2, Longyun Guo, Rohit Jaini ${ }^{3}$, Joseph H. Lynch', Rachel M. McCoy ${ }^{1}$, Jacob T. Shreve ${ }^{4, \dagger}$, Jyothi Thimmapuram ${ }^{4}$ David Rhodes ${ }^{2}$, John A. Morgan ${ }^{1,3} \&$ Natalia Dudareva ${ }^{1,2}$

In addition to proteins, L-phenylalanine is a versatile precursor for thousands of plant metabolites. Production of phenylalanine-derived compounds is a complex multi-compartmental process using phenylalanine synthesized predominantly in plastids as precursor. The transporter(s) exporting phenylalanine from plastids, however, remains unknown. Here, a gene encoding a Petunia hybrida plastidial cationic amino-acid transporter (PhpCAT) functioning in plastidial phenylalanine export is identified based on homology to an Escherichia coli phenylalanine transporter and co-expression with phenylalanine metabolic genes. Radiolabel transport assays show that PhpCAT exports all three aromatic amino acids. PhpCAT downregulation and overexpression result in decreased and increased levels, respectively, of phenylalanine-derived volatiles, as well as phenylalanine, tyrosine and their biosynthetic intermediates. Metabolic flux analysis reveals that flux through the plastidial phenylalanine biosynthetic pathway is reduced in PhpCAT RNAi lines, suggesting that the rate of phenylalanine export from plastids contributes to regulating flux through the aromatic amino-acid network.

\footnotetext{
${ }^{1}$ Department of Biochemistry, Purdue University, 175 South University Street, West Lafayette, Indiana 47907-2063, USA. ${ }^{2}$ Department of Horticulture and Landscape Architecture, Purdue University, 625 Agriculture Mall Dr, West Lafayette, Indiana 47907-2010, USA. ${ }^{3}$ School of Chemical Engineering, Purdue University, 480 Stadium Mall Dr, West Lafayette, Indiana 47907-2100, USA. ${ }^{4}$ Bioinformatics Core, Purdue University, 155 South Grant Street, West Lafayette, Indiana 47907, USA. † Present addresses: Department of Biology, Duke University, Durham, North Carolina 27708, USA (H.Y.); Indiana University School of Medicine South Bend, South Bend, Indiana 46617, USA (J.T.S.). Correspondence and requests for materials should be addressed to N.D. (email: dudareva@purdue.edu).
} 
$\mathrm{T}$ he aromatic amino acid L-phenylalanine is a vital constituent of proteins in all living organisms and serves as a versatile precursor for thousands of indispensable metabolites $^{1}$. In humans, phenylalanine is an essential amino acid obtained through the diet primarily from plant-based sources, either directly or indirectly. Humans can convert phenylalanine to L-tyrosine for incorporation into proteins, as well as to synthesize thyroid hormones ${ }^{2}$ and brain chemicals such as L-dopamine, epinephrine and norepinephrine ${ }^{3}$. In plants, phenylalanine metabolism is even more prevalent and diverse. In fact, plants direct $20-30 \%$ of photosynthetically fixed carbon to the production of phenylalanine $e^{4}$ and phenylalanine-derived compounds, which constitute approximately $30-45 \%$ of plant organic matter ${ }^{5}$ and have profound impacts on growth and development (for example, lignin ${ }^{6}$ ), reproduction (for example, phenylpropanoids and benzenoids ${ }^{7}$ ) and defence (for example, salicylic acid ${ }^{8}$, tannins and flavonoids ${ }^{9}$ ).

Production of phenylalanine-derived compounds in plants is a complex multi-compartmental process relying on phenylalanine mainly synthesized in plastid stroma ${ }^{10-12}$ to first be exported to the cytosol across both the inner and outer plastid envelope membranes. Due to its low permeability coefficient $\left(2.5 \times 10^{-10} \mathrm{~cm} \mathrm{~s}^{-1}\right.$ for phenylalanine versus $2 \times 10^{-4} \mathrm{~cm} \mathrm{~s}^{-1}$ for water in egg phosphatidylcholine $)^{13,14}$, simple diffusion of phenylalanine through membranes cannot meet the cytosolic demand, and therefore its passage is predicted to be protein mediated. In general, the plastid outer envelope is believed to contain pores and channels that confer partial discriminatory transport of metabolites, while the inner membrane, in conjunction with over 100 different transporters, serves as a selective permeability barrier ${ }^{15}$. Within the outer plastid envelope, an outer envelope protein, OEP16 (ref. 16), a member of the plant preprotein and amino-acid transporter superfamily ${ }^{17}$, forms cation-selective high-conductance channels with remarkable permeability for amines and amino acids, including phenylalanine ${ }^{18}$. However, there are still no reports on the characterization of inner envelope amino-acid transporters ${ }^{19}$, despite the presence of several amino-acid biosynthetic pathways in plastids ${ }^{20}$. Phenylalanine is utilized throughout the cell in plant primary and specialized metabolism; thus, its transport across membranes is not limited to plastids. While intercellular amino-acid transporters have been uncovered through recent studies on long-distance nitrogen transport in plants, to date very little is known about intracellular transport of amino acids ${ }^{21-24}$.

Phenylalanine transport (uptake) has been well studied in microbes. In yeast (Saccharomyces cerevisiae) phenylalanine uptake occurs via the broad substrate transporters AGP1 and GAP1, the high-affinity tryptophan permease TAT2, and the branched-chain amino-acid permeases BAP2 and BAP3 (ref. 25). In Escherichia coli, phenylalanine uptake proceeds via four distinct systems: (i) PheP (T.C. 2.A.3.1.1), a specific transport system that prefers phenylalanine over tyrosine and does not accept L-tryptophan ${ }^{26}$; (ii) AroP, a general aromatic amino-acid transport system actively transporting phenylalanine, tyrosine and tryptophan with high affinity ${ }^{27}$; (iii) TyrP, a tyrosine-specific system transporting phenylalanine at $\mathrm{mM}$, but not $\mu \mathrm{M}$, concentrations $^{28}$; and (iv) LIV-I/LS, a branched-chain aminoacid transporter system with broad substrate specificity capable of transporting phenylalanine only at very high concentrations ${ }^{29}$.

The flowers of Petunia hybrida cv Mitchell have emerged as an ideal model system for studying plant phenylalanine metabolism. The primary fate of phenylalanine in mature petunia flowers is towards production of cytosolically synthesized volatiles ${ }^{30,31}$. However, it has been shown that phenylalanine is predominantly synthesized in plastids ${ }^{10-12}$, implicating the involvement of an unknown transporter to move phenylalanine to the cytosol. Based on homology with E. coli $\mathrm{PheP}$ and coexpression with phenylalanine metabolic genes, we have identified a gene encoding a $P$. hybrida plastidial cationic amino-acid transporter (PhpCAT) that participates in plastidial phenylalanine transport. Green fluorescent protein (GFP) fusion experiments and subcellular fractionation combined with immunoblotting confirmed that PhpCAT is localized to plastids. Expression of PhpCAT was found to be spatially, developmentally and temporally consistent with genes involved in biosynthesis of phenylalanine and phenylalanine-derived volatiles. Radiolabel uptake assays performed using E. coli-overexpressing PhpCAT suggest that this transporter is capable of transporting all three aromatic amino acids. RNAi downregulation of PhpCAT resulted in reduced emission of phenylalanine-derived volatiles and lowered levels of phenylalanine and tyrosine, and to a lesser extent tryptophan. In contrast, PhpCAT overexpression led to increased flux to phenylalanine-derived volatiles and elevated aromatic amino-acid pools. Metabolic flux analysis further showed that when PhPCAT is downregulated flux is increased through the alternative phenylpyruvate route, which synthesizes phenylalanine in the cytosol. Based on these data we conclude that PhpCAT is involved in export of phenylalanine from plastids and contributes to controlling carbon flux through the phenylalanine biosynthetic network.

\section{Results}

PhpCAT is a homologue of $E$. coli pheP localized in plastids. With recent advances in gene expression technology, coexpression analysis is becoming an effective tool for discovery of missing genes, including those encoding for plastidial transporters ${ }^{32,33}$. Therefore, we took advantage of the fact that petunia flowers synthesize high levels of phenylalanine and phenylalanine-derived volatiles, production of which is coordinately regulated over development and a daily light/dark cycle $^{10,11,34-37}$. We generated an RNA-Seq data set based on RNA isolated from corolla tissue harvested at $20: 00 \mathrm{~h}$ at two developmental stages, day -1 (bud) and day 2 postanthesis (the tissues containing the lowest and highest levels of phenylalanine, respectively ${ }^{10}$ ) to accurately detect and quantify low-abundance transcripts not represented in our petunia petal-specific EST database $\mathrm{s}^{30}$ or the Sol genomics network (http://solgenomics.net). To identify candidate phenylalanine transporters, a tblastn search (cutoff $\mathrm{e}^{-08}$ ) of the petunia RNA-Seq de novo assembly transcriptome was performed for genes encoding proteins homologous to E. coli $\mathrm{PheP}^{26}$. Based on the cutoff, three distinct genes, corresponding to contigs Ph21511, Ph18042 and Ph19221, were retrieved and appeared to represent full-length mRNAs encoding proteins comprised of at least 467 amino acids that share $24 / 41 \%, 22 / 43 \%$ and $22 / 41 \%$ identity/similarity to E. coli PheP, respectively. The cognate proteins of contigs Ph21511 and Ph18042 were found to contain 14 and 13 transmembrane helices, respectively, and were annotated by BLAST2GO in the RNA-Seq data sets as putative cationic amino-acid transporters (CATs; T.C. 2.A.3.3.4), which are proton-energized members ${ }^{38}$ of the amino acid-polyamineorganocation transporter family ${ }^{15}$. Contig Ph19221 was predicted to encode for a putative amino-acid transporter with nine transmembrane domains.

In petunia, it has been shown that expression of phenylalanine metabolic genes increases concomitantly with increased phenylalanine levels ${ }^{10,11}$ and emitted volatiles ${ }^{34,35}$ as the flower opens. We hypothesized that a phenylalanine transporter may also be transcriptionally upregulated at this time. Since RNA-Seq is a quantitative approach, gene expression on day 2 after flower opening relative to buds was investigated based on the number of 
counts corresponding to each contig in the generated data sets. The counts are based on the number of reads that map to a given contig normalized for the total number of reads and length of the contig (fragments per kilobase of transcript per million mapped (FPKM) values), which were used by Cufflinks for differential gene expression. First, higher expression of genes encoding plastidial phenylalanine biosynthetic enzymes (chorismate mutase 1, CM1; prephenate aminotransferase, PPA-AT; arogenate dehydratase 1, ADT1) and cytosolic phenylalanine-utilizing enzymes involved in scent formation (phenylalanine ammonia-lyase 1, PAL1; phenylacetaldehyde synthase, PAAS) on day 2 relative to buds (Fig. 1 and Supplementary Table 1) was confirmed to validate the RNASeq data sets. Next, expression of the three petunia pheP homologues was examined. This analysis revealed that contig Ph19221 showed an average 1.8-fold decrease in counts on day 2 relative to buds, while contigs $P h 21511$ and $P h 18042$ both showed a 2-fold average increase (Fig. 1 and Supplementary Table 1). Therefore, only Ph21511 and Ph18042 were further examined as plastidial phenylalanine transporter candidate genes.

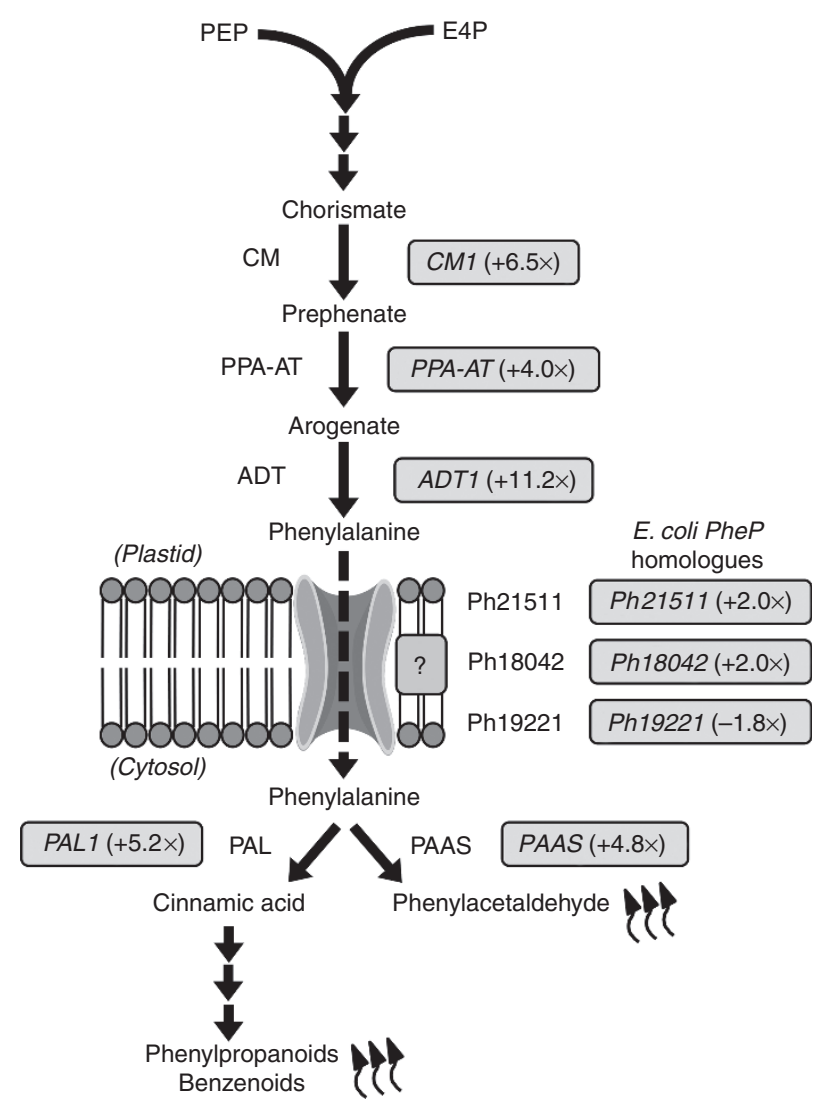

Figure 1 | Identification of plastidial phenylalanine transporter candidates. Grey boxes depict average fold change in gene expression in petunia flowers on day 2 postanthesis relative to the bud stage. Changes in expression are shown for petunia homologues of E. coli pheP, genes encoding plastidial phenylalanine biosynthetic enzymes chorismate mutase 1 (CM1), prephenate aminotransferase (PPA-AT) and arogenate dehydratase 1 (ADT1), as well as genes for the cytosolic phenylalanine-utilizing enzymes phenylalanine ammonia lyase 1 (PAL1) and phenylacetaldehyde synthase (PAAS). Measurement of gene expression is based on the average number of sequenced fragments \pm s.e.m. ( $n=3$ biological replicates) found for a given contig (gene) per kilobase of sequence per million reads (FPKM) from RNA-Seq analysis of petunia flowers collected at 20:00 $\mathrm{h}$ at the bud stage (day -1$)$ and on day 2 postanthesis.
Recent analysis of the 5,800 transmembrane proteins in Arabidopsis thaliana revealed that 660 contained putative amino-terminal plastid transit peptides ${ }^{15}$. Therefore, it was hypothesized that if contigs Ph21511 and Ph18042 encode plastidial transporters their cognate proteins should bear detectable N-terminal transit peptides. Analysis of the encoded Ph18042 protein by multiple subcellular prediction programs (WoLF PSORT: http://www.genscript.com/psort/wolf_psort.html; Predotar: https://urgi.versailles.inra.fr/predotar/predotar.html; TargetP: http://www.cbs.dtu.dk/services/TargetP/) revealed that it does not contain a transit peptide. In contrast, the first 43 amino acids of the encoded Ph21511 protein were predicted with a score of 0.837 by TargetP to serve as a plastid-targeting signal. To experimentally test for plastidial localization, the first 52 amino acids of Ph21511, which include all the residues prior to the first predicted transmembrane domain, were fused to the $\mathrm{N}$-terminus of the GFP reporter protein and transiently expressed in tobacco leaves (Fig. 2a-f). Whereas the GFP control displayed fluorescence confined to the cytosol (Fig. 2a-c), the green fluorescence of the Ph21511 1 -52-GFP construct resulted in co-localization with the red autofluorescence of chlorophyll in plastids (Fig. 2d-f). To independently verify the confocal microscopy result, total crude extracts and isolated plastids were prepared from 2-day-old petunia flowers and examined by immunoblotting using purified anti-Ph21511 antibodies. The apparent molecular mass of the detected protein, which migrated above the $50-\mathrm{kDa}$ marker, from both crude extract and the plastid fraction was consistent with the calculated size $(57.9 \mathrm{kDa})$ for mature (that is, without transit peptide) Ph21511 (Fig. 2g). Moreover, the detected signal from equal amounts

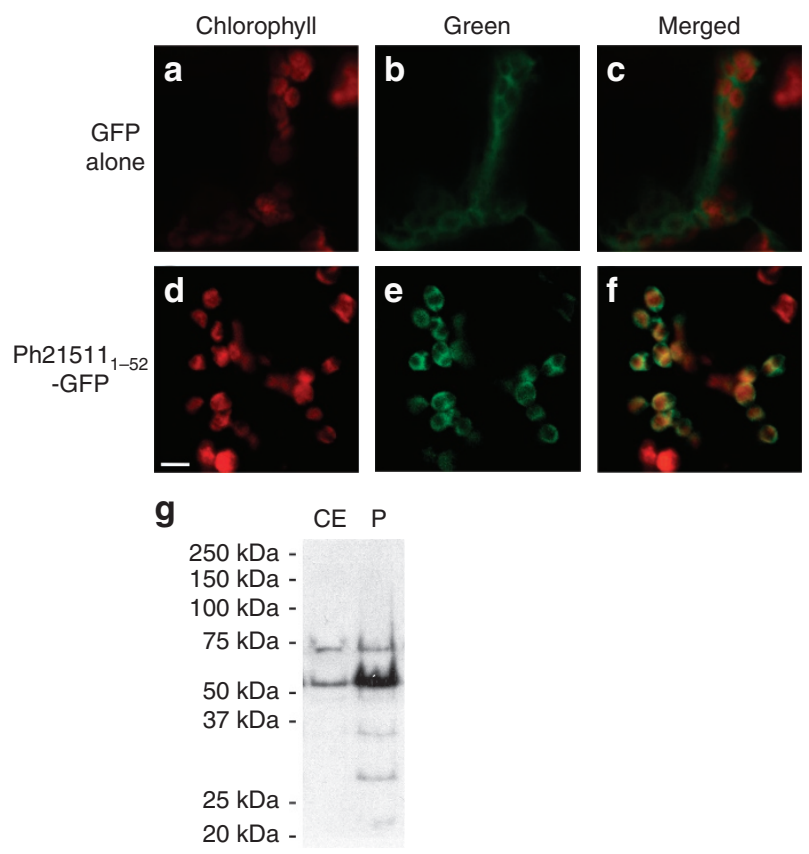

Figure 2 | Ph21511 is localized to plastids. (a-c) Transient expression of GFP alone in leaves of Nicotiana benthamiana. (d-f) Transient expression of the first 52 amino acids of Ph21511 fused to the N-terminal end of GFP in leaves of Nicotiana benthamiana. Red pseudocolour of chlorophyll autofluorescence is shown in panels $\mathbf{a}$ and $\mathbf{d}$. Green pseudocolour of GFP fluorescence is shown in panels $\mathbf{b}$ and $\mathbf{e}$. Merged images of red and green pseudocolour from $\mathbf{a}$ and $\mathbf{b}$ and from $\mathbf{d}$ and $\mathbf{e}$ are shown in $\mathbf{c}$ and $\mathbf{f}$, respectively. Scale bar, $5 \mu \mathrm{m}$. (g) Immunoblot using anti-Ph21511 antibodies against $60 \mu$ p protein from total crude extract (CE) and plastids (P) prepared from day 2 petunia flowers. 
$(60 \mu \mathrm{g})$ of loaded protein was drastically enriched in the plastid fraction compared to crude extract (Fig. $2 \mathrm{~g}$ ), thus confirming that $\mathrm{Ph} 21511$ is targeted to plastids. Based on the experimentally determined localization and the work presented below, the protein encoded by Ph21511 was designated as PhpCAT for $P$. hybrida plastidial cationic amino-acid transporter.

PhpCAT is developmentally and rhythmically regulated. Petunia flowers predominantly emit high levels of phenylalaninederived volatiles at night ${ }^{30,39}$. To accomplish this, petunia flowers have evolved synchronized developmental and temporal expression patterns for genes involved in synthesis of phenylalanine ${ }^{10,11,36}$ and phenylalanine-derived volatiles ${ }^{34,35,37}$. Investigation of PhpCAT transcript levels by quantitative reverse transcription ( $\mathrm{qRT}$ )-PCR with gene-specific primers revealed that it is expressed in all flower organs, as well as in leaves (Fig. 3a). Moreover, PhpCAT expression was lowest at the bud stage (day -1 ) and increased upon flower opening (Fig. 3b), displaying a typical pattern for petunia flower genes involved in biosynthesis of phenylalanine $e^{10,11,36}$ and phenylalanine-derived scent compounds ${ }^{34,35,37}$ (Fig. 1 and Supplementary Table 1). Remarkably, PhpCAT was also found to exhibit rhythmic expression over a daily light cycle, with the highest transcript abundance occurring at night on day 2 postanthesis (Fig. 3c), which corresponds to the time of highest emission of phenylalanine-derived volatiles ${ }^{30,39}$. Thus, the developmental
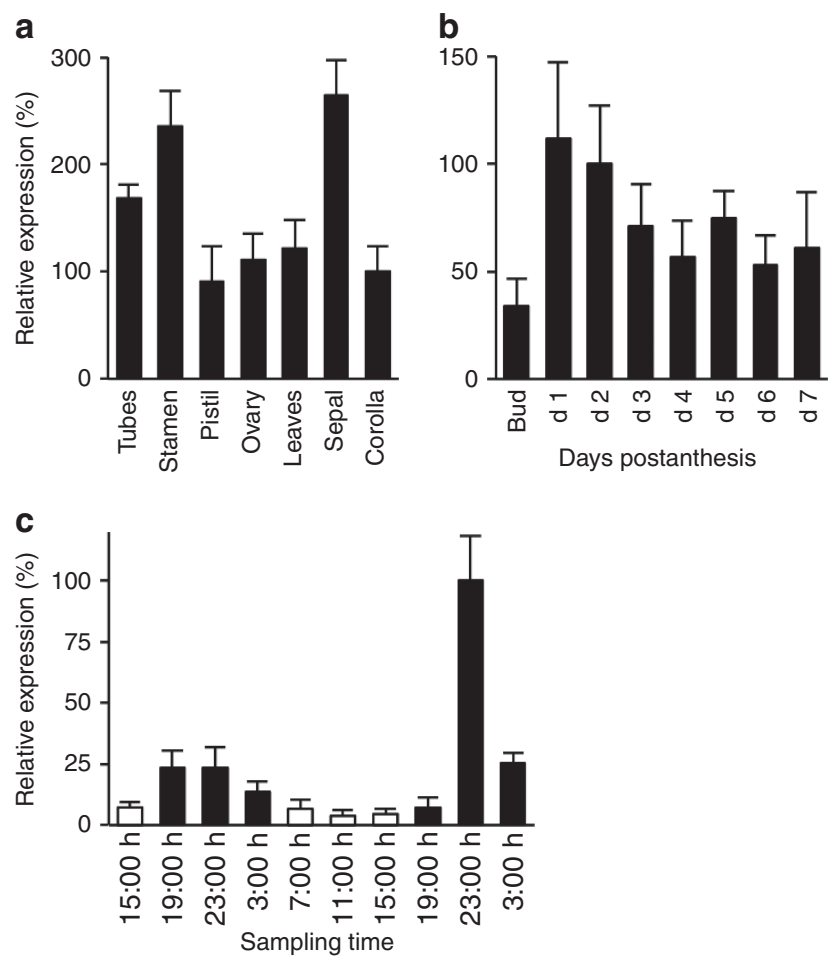

Figure 3 | Expression profiles of PhpCAT. (a) Tissue-specific expression of PhpCAT in leaves and floral organs at 15:00 h on day 2 postanthesis relative to corolla set at $100 \%$. (b) Developmental PhpCAT expression profile at 15:00 $\mathrm{h}$ in petunia flowers starting from the bud stage and on days 1 through 7 postanthesis relative to expression on day 2 set at $100 \%$.

(c) Rhythmic changes in PhpCAT expression in corollas of petunia flowers from day 1 to day 3 postanthesis during a normal light/dark cycle shown relative to expression at $23: 00 \mathrm{~h}$ on day 2 set at $100 \%$. All transcript levels were determined by qRT-PCR and obtained relative to the reference gene (elongation factor 1- $\alpha$ ). Data are means \pm s.e.m. $(n=3$ biological replicates). and temporal expression of PhpCAT is consistent with that of a transporter a priori needed to export phenylalanine out of plastids for production of floral volatiles.

PhpCAT transports all three aromatic amino acids. The use of whole-cell uptake assays in a heterologous E. coli expression system has been an informative approach for elucidating the biochemical function of plastid transporters ${ }^{40-45}$. To determine if PhpCAT is capable of transporting phenylalanine and/or the other aromatic amino acids, uptake assays with ${ }^{14} \mathrm{C}$-phenylalanine, ${ }^{14} \mathrm{C}$-tyrosine and ${ }^{14} \mathrm{C}$-tryptophan were performed with intact $E$. coli cells expressing recombinant PhpCAT $_{53-583}$ (N-terminal 6XHis-tagged PhpCAT without its predicted transit peptide) or carrying an empty vector as a control. An immunoblot using purified anti-PhpCAT (anti-Ph21511) antibodies against E. coli crude extracts verified that recombinant $\mathrm{PhpCAT}_{53-583}$ was expressed in isopropyl-1thio- $\beta$-D-galactopyranoside (IPTG)-induced cells carrying the
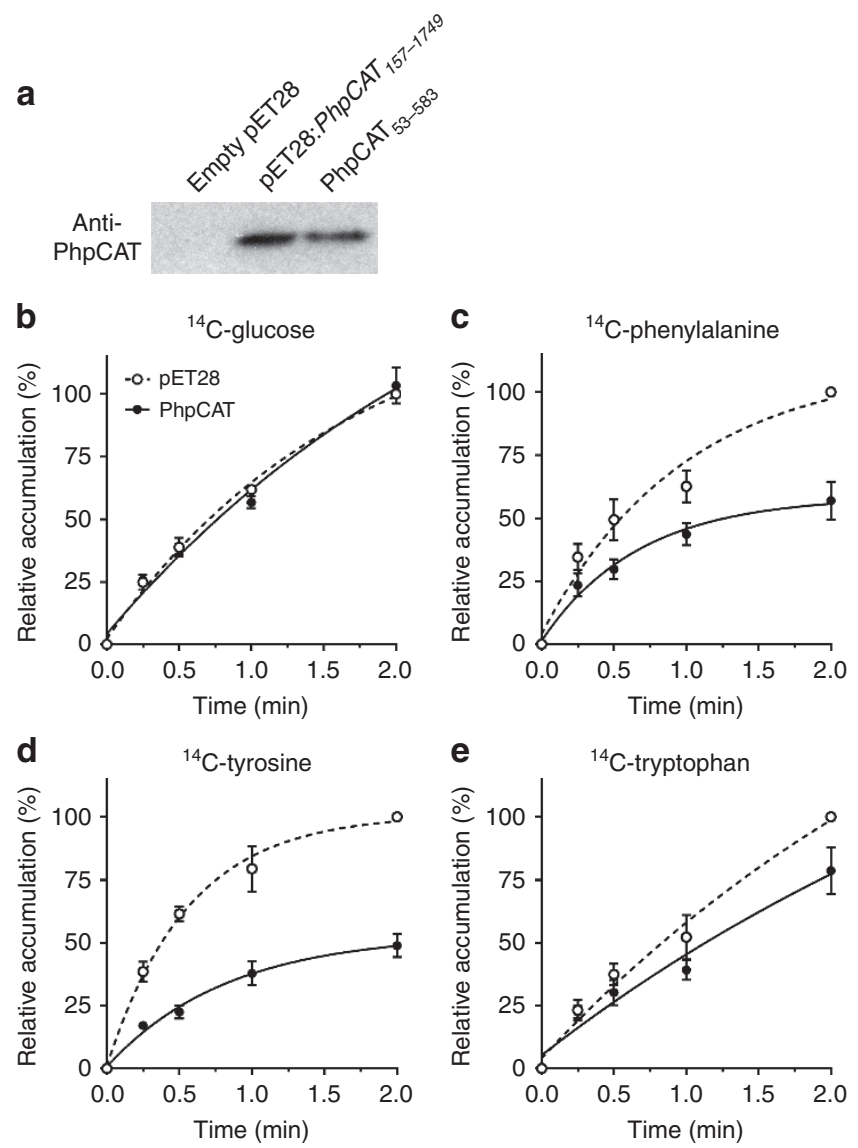

Figure 4 | PhpCAT aromatic amino-acid transport activity.

(a) Immunoblots on $2.5 \mu \mathrm{g}$ of whole-cell crude extracts prepared from E. coli carrying an empty $\mathrm{pET} 28 \mathrm{a}$ vector or codon-optimized pET28a: PhpCAT $157-1749$, and $2.5 \mu \mathrm{g}$ of purified PhpCAT ${ }_{53-583}$. (b-e) Transport assays on whole $E$. coli cells carrying empty pET28a (white circles) or pET28a:PhpCAT ${ }_{157-1749}$ (black circles). An equal number of cells were incubated with $100 \mu \mathrm{M}{ }^{14} \mathrm{C}$-glucose as a negative control (b), ${ }^{14} \mathrm{C}$-phenylalanine $(\mathbf{c}),{ }^{14} \mathrm{C}$-tyrosine $(\mathbf{d})$ or ${ }^{14} \mathrm{C}$-tryptophan (e) for the indicated time periods until termination of the assays by rapid filtration. Data are presented as a percentage relative to the corresponding empty-vector control value at 2 min set to $100 \%$. Data are means \pm s.e.m. ( $n \geq 3$ biological replicates). Two-tailed Student's $t$-tests revealed that relative accumulation of radiolabelled aromatic amino acids was statistically different in E. coli-expressing PhpCAT versus empty-vector control $(P \leq 0.01)$. 
pET28a:PhpCAT ${ }_{157-1749}$ construct, but not empty pET28a vector (Fig. 4a). As a negative control, ${ }^{14} \mathrm{C}$-glucose was used to test if overexpression of PhpCAT affected endogenous E. coli uptake activities. First, glucose was selected because it was not expected to be a substrate of PhpCAT. In addition, one of the native glucose transport systems is proton driven similar to the E. coli AroP uptake system, which is responsible for $80-90 \%$ of aromatic amino-acid transport. Since no difference in ${ }^{14} \mathrm{C}$-glucose uptake was detected between $E$. coli harbouring the empty vector and cells expressing PhpCAT $_{53-583}$, this indicates that proton-driven metabolite transport, including the endogenous aromatic amino-acid uptake system, across the membrane was not perturbed (Fig. 4b). On the other hand, E. coli cells expressing PhpCAT $\mathrm{P}_{53-583}$ accumulated significantly lower levels of ${ }^{14} \mathrm{C}$-phenylalanine (Fig. $4 \mathrm{c}$ ), ${ }^{14} \mathrm{C}$-tyrosine (Fig. $4 \mathrm{~d}$ ) and, to a lesser extent, ${ }^{14} \mathrm{C}$-tryptophan (Fig. $4 \mathrm{e}$ ), compared to the empty-vector control. The detected levels of radiolabelled amino acids result from the net difference between uptake into and efflux out of the cell. Since no decrease in uptake via the endogenous aromatic amino acid E. coli transport systems is presumably occurring, the reduced accumulation of aromatic amino acids in cells expressing PhpCAT $\mathrm{PB}_{53-583}$ is due to efflux via PhpCAT. These results demonstrate that all three aromatic amino acids are substrates of PhpCAT.

PhpCAT links plastidial-cytosolic phenylalanine metabolism. To examine if PhpCAT functions in vivo as an exporter of phenylalanine from plastids, PhpCAT expression was downregulated using an RNAi strategy under control of a petal-specific promoter ${ }^{46}$. Three independent lines with $75-80 \%$ downregulated PhpCAT transcript levels (Fig. 5a) were selected for subsequent detailed metabolic profiling. Moreover, immunoblot analysis of crude extracts and purified plastid fractions from RNAi line 17 with purified anti-PhpCAT (anti-Ph21511) antibodies indicated that PhpCAT was downregulated at the protein level (Supplementary Fig. 1). Consistent with an in planta role in plastidial phenylalanine export, downregulation of $P h p C A T$ led to $20-42 \%$ reduction in total emission of phenylalanine-derived volatiles relative to control (Fig. 5a), but the extent of decrease varied for each compound (Supplementary Fig. 2). Phenylalanine and tyrosine levels were also decreased in PhpCAT-RNAi lines by up to $42 \%$ compared to control (Fig. $5 \mathrm{a}$ ), which was likely due to reduced amounts of their biosynthetic intermediates prephenate (25-52\% decreased in PhpCAT-RNAi lines versus control) and arogenate (54-61\% decreased in PhpCAT-RNAi lines versus control) (Fig. 5a). At the same time, only minimal decreases were observed in the pool sizes of tryptophan (5-18\%

Figure 5 | Metabolic profiling of petunia flowers from PhpCAT-RNAi and PhpCAT-overexpression lines. (a) Effects of PhpCAT RNAi suppression. (b) Effects of PhpCAT overexpression. PhpCAT mRNA levels ( $n=3$ biological replicates), internal pools of shikimate, prephenate, arogenate, phenylalanine, tyrosine and tryptophan $(n=4)$, and total emission of phenylalanine-derived volatiles $(n \geq 3)$ were measured in three independent PhpCAT-RNAi downregulated and PhpCAT-overexpression lines (white bars) and compared to control values (black bars), which were set at 100\%. Data are means \pm s.e.m. ${ }^{\star} P<0.05,{ }^{\star \star} P P<0.01$ by two-tailed Student's $t$-tests. Dotted lines indicate trafficking steps. White boxes with question marks indicate unknown transporters/transport steps. In (a) the ' $x$ ' over PhpCAT depicts downregulation. In (b) the arrow with 'OX' depicts overexpression. $A D H$, arogenate dehydrogenase; $A D T$, arogenate dehydratase; $C H A$, chorismate; CM, chorismate mutase; E4P, erythrose 4-phosphate; PPA-AT, prephenate aminotransferase; PDT, prephenate dehydratase; PEP, phosphenolpyruvate; PPY-AT, phenylpyruvate aminotransferase. reduced) and shikimate (up to $9 \%$ reduced) in PhpCAT-RNAi lines compared to control (Fig. 5a).

To further investigate the role of PhpCAT in vivo, PhpCAT was overexpressed under the control of a petal-specific promoter ${ }^{44}$. Three different lines showing 4.5-100-fold increase in PhpCAT transcript levels (Fig. 5b) were chosen for further metabolite analysis. Total emission of phenylalanine-derived volatiles in detached flowers increased by up to $18 \%$ in $P h p C A T$ overexpressors compared to control (Fig. 5b), although the degree of increase differed for individual compounds (Supplementary Fig. 3). In addition, the levels of phenylalanine
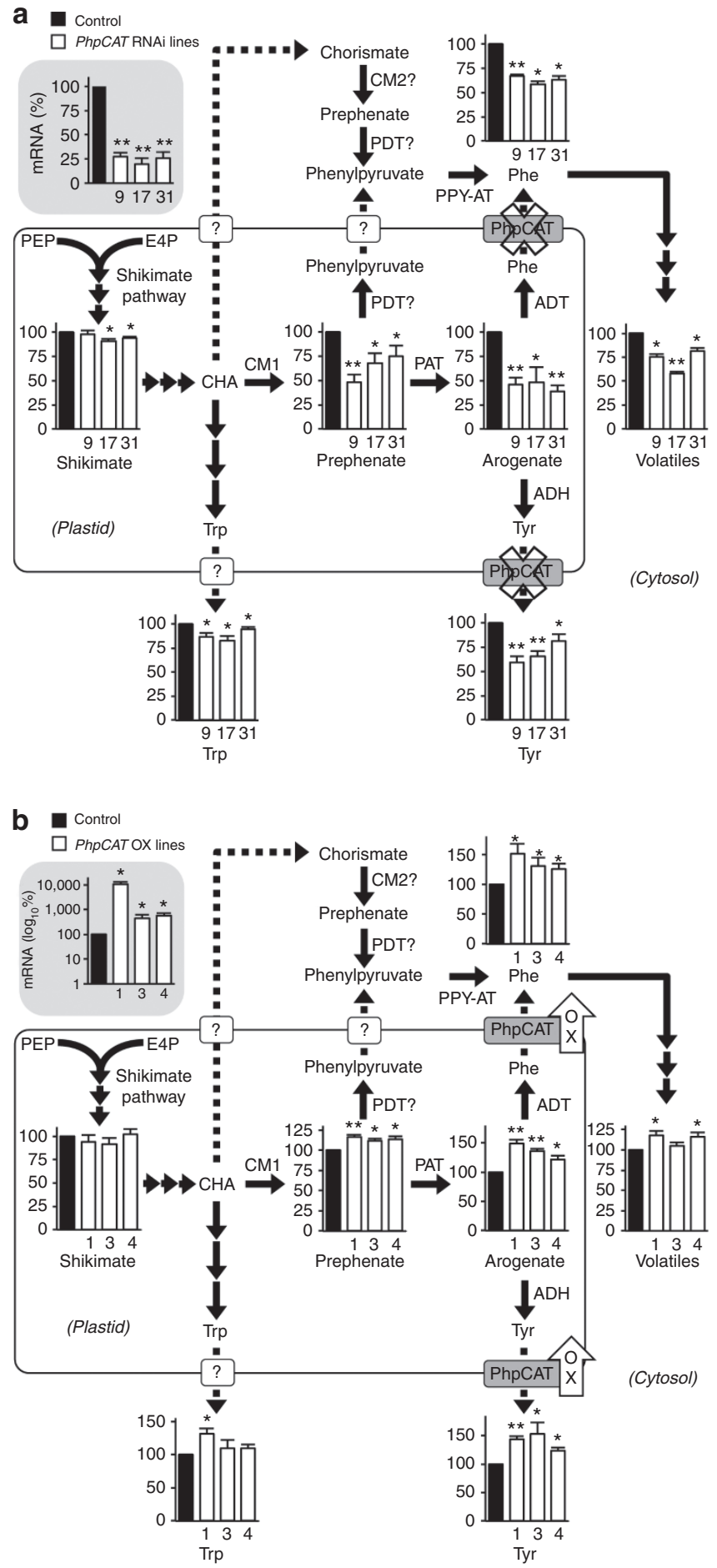
and tyrosine increased from 23 to $53 \%$ in PhpCAT overexpressors compared to control (Fig. 5b), likely as a result of elevated pools of phenylalanine biosynthetic intermediates prephenate and arogenate $(11-16 \%$ and $22-48 \%$ increase in PhpCAT overexpressors versus control, respectively) (Fig. 5b). Moreover, the tryptophan pool displayed an increasing trend and no changes were detected in shikimate levels in PhpCAT overexpressors compared to control (Fig. 5b). Taken together, these data indicate that PhpCAT is involved in plastidial phenylalanine export in planta. Moreover, profiling of plastidial phenylalanine biosynthetic intermediates suggests that PhpCAT is involved in regulating flux through the aromatic amino-acid biosynthetic network.

PhpCAT controls phenylalanine biosynthetic flux. Since downregulation of PhpCAT led to a decrease in the levels of phenylalanine, tyrosine, and their shared precursors prephenate and arogenate (Fig. 5a), we hypothesized that phenylalanine and tyrosine accumulate inside plastids of PhpCAT-RNAi lines and feedback inhibit the arogenate pathway (Fig. 6a). Recently we showed that plants also contain an alternative pathway that proceeds via phenylpyruvate to produce phenylalanine in the cytosol, and flux through this route increases when the plastidial biosynthetic pathway (via arogenate) is impaired ${ }^{12}$. In the alternative pathway, a cytosolic phenylpyruvate aminotransferase preferentially converts phenylpyruvate to phenylalanine using tyrosine as an amino donor ${ }^{12}$ (Fig. 6a). Interestingly, tyrosine cannot serve as an amino donor for PPA-AT in the plastidal arogenate pathway ${ }^{11}$. Taking advantage of this characteristic to distinguish between the two pathways, we employed metabolic flux analysis with stable isotopic labelling using ${ }^{15} \mathrm{~N}$-tyrosine to determine the effect of reduced plastidial phenylalanine export on carbon flux through the parallel phenylalanine biosynthetic pathways.

Excised 2-day-old petunia flowers from control and PhpCAT-RNAi lines 9 and 17 were fed with $10 \mathrm{mM}^{15} \mathrm{~N}$-tyrosine starting at 18:00 h, harvested after 2, 4 and $6 \mathrm{~h}$, and analysed by liquid chromatography-mass spectrometry (LC-MS) to determine phenylalanine and tyrosine pool sizes and isotope abundances. Similar to what was observed previously ${ }^{12}$, the labelling percentage and concentration of phenylalanine increased linearly over $6 \mathrm{~h}$, as did the level of emitted volatiles (Supplementary Fig. 4). To assess the control PhpCAT exerts on metabolic fluxes through the phenylalanine biosynthetic network, a metabolic flux model was developed (see Methods). The simulation revealed that $v_{1}$, the rate of synthesis through the plastidial arogenate pathway, was 32 and $44 \%$ lower $(P<0.05$, Student's $t$-test, $n \geq 3)$ at $t_{0}$ and $t_{6}$, respectively, in PhpCAT-RNAi lines compared to control (Fig. $6 \mathrm{~b}$ and Supplementary Table 2). This finding is consistent with our hypothesis that reduced export of phenylalanine and tyrosine from plastids in PhpCAT-RNAi lines leads to feedback inhibition of the arogenate pathway. At the same time, the flux analysis showed that in control and the PhpCAT-RNAi lines, $v_{2}$, the flux through the cytosolic phenylpyruvate pathway, was minor at $t_{0}$, but significantly increased over the 6-h period (Fig. $6 \mathrm{~b}$ and Supplementary Table 2). In addition, increase in the rate of $v_{2}$ in PhpCAT knockdowns was more rapid than in control $(P<0.01$, Student's $t$-test, $n \geq 3$ ) (Fig. $6 \mathrm{~b}$ and Supplementary Table 2), suggesting that more carbon flux is directed through the cytosolic pathway. Taking this into account with the decrease in flux through the arogenate pathway, the relative contribution of cytosolic phenylalanine production is considerably higher in PhpCAT-RNAi lines compared to control (a $v_{2} / v_{1}$ flux ratio of 0.44 versus 0.18 , respectively, at $t_{6}$ ) (Fig. $6 \mathrm{~b}$ and Supplementary Table 2).

\section{Discussion}

Eukaryotic metabolic networks are often spread throughout multiple subcellular compartments separated by organellar membrane barriers, through which precursors, intermediates and end products must pass. While some metabolites may passively diffuse across membranes, others require protein-mediated transport, as is the case for phenylalanine synthesized in plastids. The current study took advantage of the distinctive transcriptional developmental regulation of phenylalanine metabolism in petunia flowers to identify a plastidial amino-acid transporter, PhpCAT, which is capable of transporting all three aromatic amino acids (Fig. 4). Increases and decreases in the levels of phenylalaninederived volatiles, as well as in phenylalanine, tyrosine, and their biosynthetic intermediates prephenate and arogenate in $P h p C A T$ overexpression and RNAi lines, respectively (Fig. 5), support an in vivo role for PhpCAT as a plastidial aromatic amino-acid exporter. Moreover, flux through the phenylpyruvate pathway, which produces phenylalanine in the cytosol, was increased when PhpCAT was downregulated (Fig. 6b).

It is likely that while the total cellular pools of phenylalanine and tyrosine decrease in PhpCAT-RNAi lines compared to control (Fig. 5a), these amino acids accumulate inside the plastid and feedback inhibit the shikimate and/or aromatic amino-acid biosynthetic pathways. Disproportional effects on the concentrations of phenylalanine, tyrosine, and their precursors prephenate and arogenate compared to shikimate and tryptophan (Fig. 5a) may point to feedback inhibition of CM1 (Fig. 6a) in PhpCATRNAi lines. Previously it was shown that petunia CM1 activity is not inhibited at the same concentration $(50 \mu \mathrm{M})$ of phenylalanine and tyrosine $\mathrm{e}^{36}$ known to regulate Arabidopsis CM1 (ref. 47) However, in light of the high demand for phenylalanine in petunia flowers, and the fact that inhibition constants $\left(K_{\mathrm{i}}\right)$ of other plant CMs are in the range of $0.3-1.1 \mathrm{mM}^{1}$, it is possible that petunia CM1 is inhibited at higher concentrations of phenylalanine. To test this, we assayed preparations of petunia plastids for CM activity in the presence of a range of phenylalanine concentrations (Supplementary Figs 5 and 6). Indeed, petunia plastidial CM activity was found to be inhibited by phenylalanine with an $\mathrm{IC}_{50}$ of $748 \mu \mathrm{M}$ (Supplementary Fig. 6). Thus, the metabolic phenotype on the arogenate pathway observed in PhpCAT-RNAi lines (Fig. 5a) could be attributed to feedback inhibition of petunia CM1. Conversely, in PhpCAT- overexpression lines, levels of phenylalanine, tyrosine, prephenate and arogenate were increased compared to control (Fig. 5b), which may indicate reduced levels of phenylalanine and tyrosine in plastids leading to the relaxation of naturally occurring feedback regulation of their biosynthetic enzymes, including CM1. The fact that tryptophan also trended to increase in PhpCAT overexpression lines compared to control (Fig. 5b) might further imply reduced feedback restrictions on tryptophan biosynthesis and/or the shikimate pathway.

Downregulation of PhpCAT expression in petunia flowers by $75-80 \%$ led to $20-42 \%$ reduction in the emission of phenylalaninederived volatiles (Fig. 5a). The smaller reduction in volatiles relative to PhpCAT expression is likely the result of one or a combination of several factors. (i) It is possible that the remaining amount of PhpCAT transporter activity was sufficient to sustain the observed volatile emission rate. (ii) In addition or alternatively, there could be other specific and/or non-specific amino-acid transporters, which are not homologous to E. coli PheP, involved in exporting phenylalanine from plastids. (iii) Another possibility is the contribution of a phenylalanine storage pool in the vacuole to the production of phenylalanine-derived products. Vacuoles, 
a

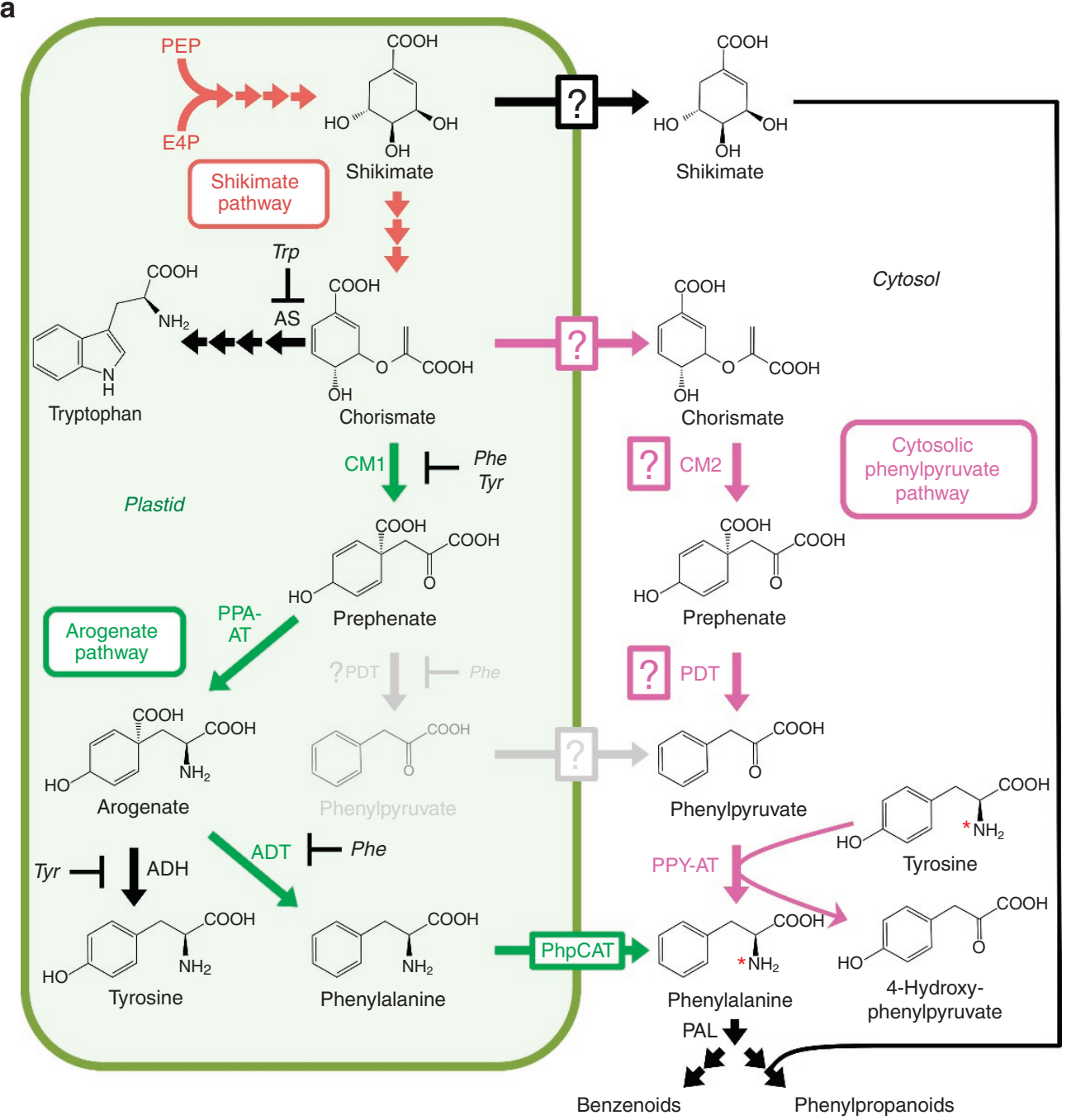

b
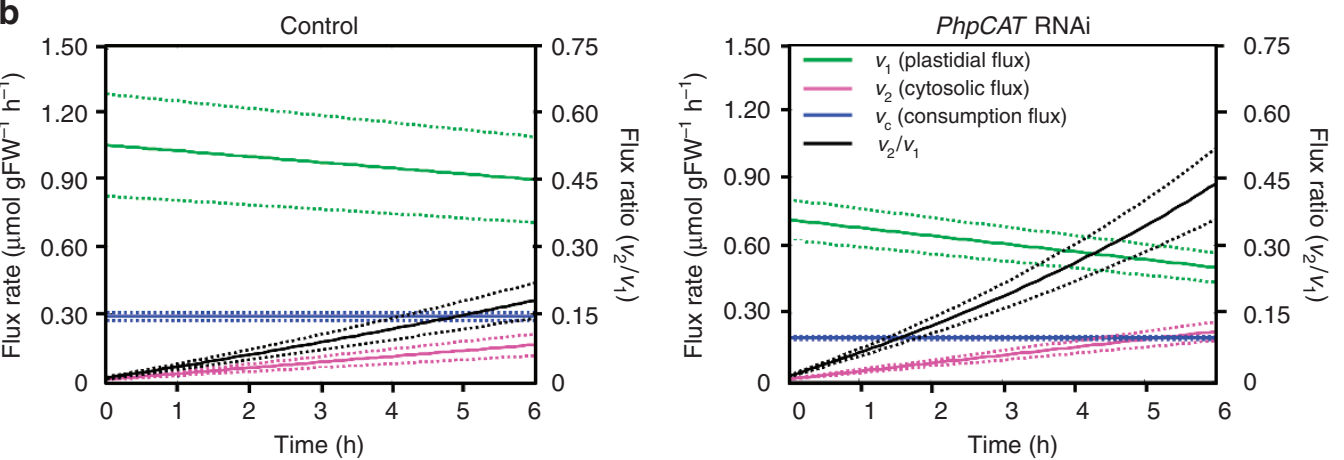

Figure 6 | Metabolic modelling of the phenylalanine biosynthetic network in control and PhpCAT-RNAi lines. (a) Scheme depicting cytosolic formation of phenylalanine and potential feedback inhibition mechanisms involved in plastidial phenylalanine biosynthesis ${ }^{1}$. In PhpCAT-RNAi lines, phenylalanine and tyrosine may accumulate in plastids and feedback inhibit arogenate dehydratase (ADT) and arogenate dehydrogenase (ADH), respectively, as well as chorismate mutase 1 (CM1). If plastidial ADTs also function as prephenate dehydratases (PDTs), then the enzymatic conversion of prephenate to phenylpyruvate may also be subject to feedback inhibition by phenylalanine. It is unclear if phenylpyruvate utilized in the alternative phenylalanine biosynthetic pathway originates from the plastid, or from cytosolic conversion of chorismate exported from the plastid. White boxes with question marks indicate unknown transporters/transport steps. AS, anthranilate synthase. For remaining abbreviations, see Fig. 5. (b) Flux models representing the phenylalanine biosynthetic network in 2-day-old petunia flowers from control and PhpCAT-RNAi lines. Computer-assisted metabolic modelling was performed using pool sizes and isotopic abundances of phenylalanine and tyrosine, and measurements of phenylalanine-derived volatile emission from petunia petal tissue supplied with $10 \mathrm{mM}{ }^{15} \mathrm{~N}$-tyrosine for up to $6 \mathrm{~h}$. The rate of plastidial flux $\left(v_{1}\right)$ in PhpCAT-RNAi lines was found to be significantly lower than control based on comparisons of absolute fluxes and two-tailed Student's $t$-tests corrected by Bonferroni method $(P<0.05)$. The rate of change in cytosolic flux $\left(v_{2}\right)$ in PhpCAT-RNAi lines was found to be significantly higher than control $(P<0.01)$ based on paired-sample two-tailed Student's $t$-tests of slopes (see Methods for additional details). $n=3$ for control and $n=6$ for PhpCAT-RNAi lines. 
which are separated from cytosol by a single, semi-permeable membrane called the tonoplast, are involved in temporary and long-term storage of numerous metabolites ${ }^{48}$, including free phenylalanine ${ }^{49}$. In recent years, tonoplast-localized transporters have been discovered in plants and shown to be an integral part of a complex cellular network controlling plant metabolism ${ }^{48}$. Two Arabidopsis amino-acid transporters, AtCAT2 and AtCAT4, have been shown to localize to the vacuolar membrane ${ }^{22,23}$. Though the physiological function of AtCAT4 is still unknown, AtCAT2 has been shown to regulate free amino-acid levels in Arabidopsis leaves ${ }^{22}$. Interestingly, AtCAT2 is the most similar Arabidopsis CAT homologue to Ph18042 (Supplementary Fig. 7), the other E. coli PheP homologue with increased gene expression in petunia flowers on day 2 postanthesis (Fig. 1 and Supplementary Table 1). Similar to AtCAT2, Ph18042 is also predicted to localize to the vacuole and it seems conceivable that it may contribute to modulating cytosolic phenylalanine levels in petunia flowers. (iv) Finally, in PhpCAT-RNAi lines the phenylpyruvate pathway could partially compensate for the shortage of phenylalanine being exported from the plastid. Recently it was shown that flux through the cytosolic phenylpyruvate pathway (Fig. 6a) increases when entry into the arogenate pathway is genetically blocked ${ }^{12}$. Similarly, the present study shows that the phenylpyruvate pathway has higher relative contribution when plastidial phenylalanine export is impeded in PhpCAT-RNAi lines (Fig. 6b). It still remains an open question at which upstream step flux is redirected towards the phenylpyruvate pathway. It is possible that CM1 is feedback inhibited by phenylalanine and tyrosine accumulated in plastids of PhpCAT-RNAi lines; thus, flux may be redirected at the level of chorismate (Fig. 6a). Plants contain a cytosolic isoform of CM, $\mathrm{CM} 2$, with higher affinity for chorismate than their plastidial CM1 counterparts $^{1}$, which may produce prephenate in the cytosol for the alternative phenylalanine biosynthetic pathway (Fig. 6a). This scenario implicates the involvement of a plastidial chorismate transporter and cytosolic prephenate dehydratase (PDT), neither of which has been discovered. Alternatively, phenylpyruvate may be generated in the plastid via dehydration/decarboxylation of prephenate by moonlighting ADT (or an unidentified PDT) and then exported through an unknown transporter to the cytosol (Fig. 6a). However, PhpCAT-RNAi lines have reduced prephenate levels and the catalytic efficiency of PPA-AT ${ }^{11}$ is much higher than those of the known ADTs with moonlighting PDT activity ${ }^{10}$. Thus, together these factors likely preclude formation of a sufficient quantity of phenylpyruvate in plastids to sustain the cytosolic pathway.

The occurrence of a plastidial CAT transporter does not appear to be unique to petunia. Phylogenetic reconstruction with the Arabidopsis CAT family reveals that the closest homologues of PhpCAT are CAT7 (73\% identity/85\% similarity) and AtCAT6 (73\% identity/84\% similarity) (Supplementary Fig. 7). While Arabidopsis CAT7 has unknown function, it possesses a putative transit peptide predicted to serve as a plastid-targeting signal ${ }^{23}$. On the other hand, AtCAT6 has previously been shown to function in sink tissues as a transporter of amino acids, including phenylalanine ${ }^{38}$. Fusion of GFP to the N-terminus of AtCAT6 revealed that it is localized to the plasma membrane ${ }^{38}$. However, it should be further investigated if AtCAT6 is dual localized, because similar to Arabidopsis CAT7 and PhpCAT, it contains a predicted transit peptide ${ }^{23}$ (with a TargetP score of 0.949).

At the cellular level, transporters are integral parts of metabolic networks as they mediate interactions between multiple pathways across different subcellular compartments. Metabolic flux analysis performed in the current study strongly supports the idea that transporters, such as PhpCAT, further exert control over fluxes through metabolic networks by influencing organellar metabolite concentrations, which in turn biochemically regulate enzymes. Thus, development of organellar metabolite-profiling techniques ${ }^{50,51}$, coupled with stable isotope labelling, will be needed to further dissect the role PhpCAT plays in feedback control of the aromatic amino-acid network.

\section{Methods}

Chemicals andreagents. $\mathrm{D}-\left[{ }^{14} \mathrm{C}(\mathrm{U})\right]$ glucose $\left(300 \mathrm{mCi} \mathrm{mmol}^{-1}\right), \mathrm{L}-\left[{ }^{14} \mathrm{C}(\mathrm{U})\right]$ phenylalanine $\left(350 \mathrm{mCi} \mathrm{mmol}^{-1}\right), \mathrm{L}-\left[{ }^{14} \mathrm{C}(\mathrm{U})\right]$ tyrosine $\left(482 \mathrm{mCi} \mathrm{mmol}^{-1}\right)$ and $\mathrm{L}$-[side chain-3- ${ }^{14} \mathrm{C}$ ] tryptophan $\left(55 \mathrm{mCi} \mathrm{mmol}^{-1}\right)$ were from American Radiolabeled Chemicals (St Louis, MO). All other chemicals were from Sigma-Aldrich (http://www.sigmaaldrich.com) unless otherwise noted.

Plant material and growth conditions. Wild-type and transgenic P. hybrida cv. Mitchell diploid plants (W-115; Ball Seed Co., West Chicago, IL) were grown under standard greenhouse conditions ${ }^{44}$ with a light period from 18:00 until 21:00 h. For the PhpCAT-RNAi construct, DNA containing two spliced PhpCAT cDNA fragments of the coding region corresponding to nucleotides 30-577 and 30-368, the latter in antisense orientation to create a hairpin structure, was synthesized (Genscript, Piscataway, NJ). 5'-EcoRI and $3^{\prime}$-BamHI sites were added for directional subcloning into pRNA69 containing the Clarkia breweri linalool synthase (LIS) petal-specific promoter ${ }^{44}$. The resulting cassette containing the LIS promoter and the synthetic PhpCAT hairpin fragment was cut out using SacI/NotI and subcloned into the pART27 simpler binary vector ${ }^{52}$. For the $P h p C A T$ overexpression construct, $P h p C A T$ was amplified using the forward primer 5'-GAATTCATGGAGACCCATAGCTCCTCTTTCTCTAACATAAA- $3^{\prime}$ and reverse primer $5^{\prime}$-GGATCCTTACACTTTGAGAGTATGGTCCTGGT TTTCAATAG- $3^{\prime}$ and directionally subcloned into the EcoRI and BamHI sites (underlined in the respective primers) of pRNA69 in frame with the LIS promoter The resulting cassette was cut out with SacI/NotI and subcloned into the pART27 binary vector. The final PhpCAT-RNAi and PhpCAT overexpression constructs were used for Agrobacterium tumefaciens (strain GV3101)-mediated transformation of $P$. hybrida cv. Mitchell diploid using the standard leaf disk transformation method ${ }^{53}$

Generation of RNA-Seq data sets. RNA was isolated from corollas of at least eight wild-type P. hydrida cv. Mitchell flowers (per biological replicate) at 20:00 h on day - 1 (bud) and day 2 postanthesis using an RNeasy plant mini kit (Qiagen, Valencia, CA). RNA was treated with DNase I to eliminate genomic DNA using the TURBODNA-free kit (Ambion, Foster City, CA). A total of six cDNA libraries, representing three biological replicates of each developmental stage, were constructed using an mRNA-Seq Kit (Illumina, San Diego, CA), and 100-bp pairedend reads were generated using Illumina HiSeq2500 at the Purdue Genomics Center, with at least 35 million reads per library. Sequences were first assessed for quality by FastQC (v. 0.10.0; http://www.bioinformatics.babraham.ac.uk). Trimming was done using FASTX toolkit (v. 0.0.13; http://hannonlab.cshl.edu/fastx_toolkit/), parameters were configured to trim reads less than Phred 33 score of 30 and resulting reads had to be at least 50 bases long to be retained. After quality trimming, de novo assembly of the transcriptome was performed using Trinity ${ }^{54}$ (v. R 2012-10-05) with default parameters. Trinity generated 100,419 unique contigs ( $\geq 500 \mathrm{bp}$ ), which were then used as reference transcriptome for RNA-seq analysis and were annotated using BLAST2 GO ${ }^{55}$ (version 2.5.0) using default parameters. The N50 for these contigs was $2,509 \mathrm{bp}$, which was calculated by identifying that contig length in which $50 \%$ of the assembly's total bases are included in contigs of equal or greater length, and $50 \%$ of the assembly's total bases are included in contigs of lesser length. A total of 25,603 contigs were $\geq N 50$ (Supplementary Fig. 8). The quality-trimmed reads were mapped against the generated transcriptome using Tophat (v. 2.0.6; http://tophat.cbcb.umd.edu/) with default parameters. The total number of reads from all samples that unambiguously mapped to a gene feature was $217,939,059$ $(93.34 \%)$. Each assembled contig in the de novo transcriptome assembly was treated as a putative gene, and therefore gene counts for differential expression analysis were aggregated based on contig counts, using HTSeq (v.0.5.3p7; http://wwwhuber.embl.de/users/anders/HTSeq/). Counts for each sample were merged using custom perl scripts and used for downstream differential gene expression analysis. Although we acknowledge FPKM values may be affected, we did not attempt to collapse or reduce contigs prior to read mapping and differential gene expression analysis in order to avoid potential false negatives.

Differential expression analysis between samples 'bud' and 'day2' was performed using three different methods, of which two were using ' $R$ ' (v. 2.15.2; http://www.r-project.org/). Basic exploration of the read count data file was performed to ensure data quality. An edgeR object was created by combining the counts matrix, library sizes and experimental design using edgeR (v. 3.0.3) package ${ }^{56}$. Normalization factors were calculated for the counts matrix, followed by estimation of common dispersion of counts. Exact test for differences between the negative binomial distribution of counts for the two experimental conditions resulted in finding differential expression, which was then adjusted for multiple hypothesis testing. Another method named 'voom' from limma package ${ }^{57}$ (v. 3.14.1) was also used for differential expression analysis. The function of 'voom' carries out $\log _{2}$ transformation of counts followed by mean-variance estimation 
and assigning weight to each transformed value. Linear model coefficients were then calculated using limma's design matrix, contrast matrix and $\log _{2}$ transformed values. Linear model was fitted using empirical Bayes method and differences between counts for two experimental conditions were calculated, which were then adjusted for multiple hypothesis testing. Additionally, mapping of each sample to the transcriptome using TopHat resulted in .bam files, which were then used by the Cufflinks (v 2.0.2) suite of programs ${ }^{58}$. In all, $96.98 \%$ of the contigs found to be differentially expressed by Cufflinks were also differentially expressed by both edgeR and voom.

qRT-PCR. Sample collection, RNA isolation and qRT-PCR were performed as previously described ${ }^{35}$. Briefly, samples were collected from the tissues indicated in the text and RNA was isolated using the RNeasy Plant Mini Kit (Qiagen). Total RNA was treated with DNaseI to eliminate genomic DNA using the TURBO DNA-free Kit (Ambion) and was reverse-transcribed to cDNA using the High Capacity cDNA Reverse Transcription Kit (Applied Biosystems). PhpCAT was amplified using the specific primers $5^{\prime}$-AGCACTTTCCGATACCCCAA- $3^{\prime}$ and $5^{\prime}$-GAACTCGGCT TGTTTCTTCGA- $3^{\prime}$. PhpCAT expression was analysed relative to the reference gene elongation factor $1-\alpha$ which was amplified using primers $5^{\prime}$-CCTGGTCAAAT TGGAAACGG- $3^{\prime}$ and $5^{\prime}$-CAGATCGCCTGTCAATCTTGG- $3^{\prime}$. Each data point represents an average of three independent biological samples.

Subcellular localization of PhpCAT. The first 156 nucleotides encoding the 52 amino acids preceding the first predicted transmembrane domain of PhpCAT (Ph21511) were amplified using the forward primer 5'-CACCATGGAGACCC ATAGCTCCTCTTTCTCTAACATAAA- $3^{\prime}$ and reverse primer $5^{\prime}$-CCGTTTCATA TCACCACCAGACCG- $3^{\prime}$. The resulting fragment was subcloned into $\mathrm{PENTR}^{\mathrm{TM}}$ / $\mathrm{D}-\mathrm{TOPO}$ (Invitrogen ${ }^{\mathrm{TM}}$, Carlsbad, CA), sequence verified and transferred into pK7FWG $2^{59}$ via Gateway ${ }^{\mathrm{TM}}$ technology, resulting in an in-frame fusion with the $\mathrm{N}$-terminal end of GFP. The $\mathrm{Ph} 21511_{1-52}$-GFP construct and an empty GFP vector were transformed into A. tumefaciens strain GV3101, infiltrated into Nicotiana benthamiana leaves, and imaged $48 \mathrm{~h}$ later. Images were acquired using a Zeiss LSM710 laser spectral scanning confocal microscope with a C-Apochromat $40 \times / 1.20 \mathrm{~W}$ objective (Zeiss, Thornwood, NY). GFP was excited with an argon laser at wavelength $488 \mathrm{~nm}$ and emissions were collected over a $493-598-\mathrm{nm}$ bandpass. Chlorophyll fluorescence was excited by a HeNe laser at wavelength $633 \mathrm{~nm}$ and emissions were collected over a 647-721-nm bandpass.

\section{Heterologous expression of PhpCAT and transport assays. The coding} sequence of PhpCAT was synthesized (Genscript, Piscataway, NJ) with codon optimization for expression in E. coli using GenScript's OptimumGene ${ }^{\mathrm{TM}}$ Gene Design system. A truncated version (minus the predicted transit peptide) of codon-optimized PhpCAT was amplified with the forward primer 5'-CATATGAG TCTGCGTTGGTACGACCTGGTT- $3^{\prime}$ and reverse primer ${ }^{\prime}$-CTCGAGTTAGAC

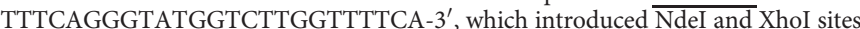
(underlined), respectively, for directional cloning into pET28a (Novagen) in frame with an N-terminal 6XHis-tag. The resulting codon-optimized pET28a: $P h p C A T_{157-1749}$ construct was sequence verified and transformed into Lemo21 (DE3) E. coli (New England Biosciences, Ipswich, MA). Starter cultures containing kanamycin and chloramphenicol were used to inoculate $25 \mathrm{ml}$ of Luria-Bertani (LB) at an $A_{600}$ of 0.05 . When $A_{600}$ reached approximately 0.4, IPTG was added to a final concentration of $0.5 \mathrm{mM}$ and incubation was continued for $2 \mathrm{~h}$ at $18^{\circ} \mathrm{C}$. Cells were harvested by centrifugation, and nickel-affinity purification of $\mathrm{PhpCAT}_{53-583}$ under denaturing conditions was performed according to The QIAexpressionist ${ }^{T M}$ (5th edn, 2003) protocols 10 and 17.

For the radiolabel uptake assays, IPTG induction and preparation of cells carrying empty pET28a or codon-optimized pET28a:PhpCAT ${ }_{157-1749}$ was performed as described above except that cells were washed and resuspended to an $A_{600}$ of 4.0 in M9 minimal media. To initiate the assays, $100 \mu \mathrm{l}$ of prepared cells was added to $100 \mu$ l of M9 incubation media containing ${ }^{14} \mathrm{C}$-labelled glucose $(0.25 \mu \mathrm{Ci})$, phenylalanine $(0.35 \mu \mathrm{Ci})$, tyrosine $(0.35 \mu \mathrm{Ci})$ or tryptophan $(0.2 \mu \mathrm{Ci})$.

Concentrations of substrates were pre-adjusted in the M9 incubation media to $200 \mu \mathrm{M}$ with cold substrate. Uptake assays were carried out at $30^{\circ} \mathrm{C}$ in $1.5 \mathrm{ml}$ Eppendorf microfuge tubes and terminated after the indicated time periods by rapid filtration of the reaction through a $0.45-\mu \mathrm{m}$ Whatman filter (GE Healthcare Life Sciences, Pittsburgh, PA) under vacuum. Filters were washed two times with $1 \mathrm{ml} \mathrm{M9}$ media and then transferred into a 20-ml scintillation vial containing $10 \mathrm{ml}$ of EcoLite (+) scintillation cocktail (MP Biochemicals, Santa Ana, CA).

Radioactivity in the samples was quantified by scintillation counting.

Metabolic flux analysis with ${ }^{15} \mathrm{~N}$-tyrosine labelling. We generated a metabolic flux model that utilizes experimentally determined pool sizes and isotopic enrichment of phenylalanine from exogenously fed ${ }^{15} \mathrm{~N}$-tyrosine. Feeding of $10 \mathrm{mM}{ }^{15} \mathrm{~N}$-tyrosine (Cambridge Isotope Laboratories, Andover, MA) to 2-day-old corollas of control and PhpCAT-RNAi lines was performed as previously described $^{12}$. Similar to our previous labelling study ${ }^{12}$, the labelling percentage of tyrosine quickly reached $>80 \%$ and a pseudo-steady state within $2 \mathrm{~h}$ upon feeding, and stayed constant for the rest of the experimental period. The model was based on the dynamic mass balance around phenylalanine as defined as

$$
\frac{\mathrm{d} C_{\mathrm{Phe}}}{\mathrm{d} t}=v_{1}+v_{2}-v_{\mathrm{c}}
$$

where $C_{\text {Phe }}$ is the phenylalanine pool size, $v_{1}$ is the flux through the plastidial arogenate pathway, $v_{2}$ is the flux through the cytosolic phenylpyruvate pathway and $v_{c}$ is the consumption rate of phenylalanine. To determine $v_{2}$, it was also required to know the mass balance of isotopic enrichment for phenylalanine as defined as

$$
\frac{\mathrm{d} f_{\text {Phe }} C_{\text {Phe }}}{\mathrm{d} t}=f_{\text {Tyr }} \times v_{2}-f_{\text {Phe }} \times v_{\mathrm{c}}
$$

where $f_{\text {Phe }}$ and $f_{\text {Tyr }}$ represent isotopic abundance of phenylalanine and tyrosine, respectively, in the total pools. With equations (1) and (2), $v_{1}$ and $v_{2}$ can be derived as follows:

$$
v_{1}=\frac{\left(f_{\text {Tyr }}-f_{\text {Phe }}\right) \frac{\mathrm{d} C_{\text {Phe }}}{\mathrm{d} t}-C_{\text {Phe }} \frac{\mathrm{d} f_{\text {phe }}}{\mathrm{d} t}}{f_{\text {Tyr }}}+\frac{f_{\text {Tyr }}-f_{\text {Phe }}}{f_{\text {Tyr }}} v_{\mathrm{c}}
$$

and

$$
v_{2}=\frac{f_{\text {Phe }} \frac{\mathrm{d} C_{\text {Phe }}}{\mathrm{d} t}+C_{\text {Phe }} \frac{\mathrm{d} f_{\text {phe }}}{\mathrm{d} t}}{f_{\text {Tyr }}}+\frac{f_{\text {Phe }}}{f_{\text {Tyr }}} v_{\mathrm{c}}
$$

Since the labeling percentage and concentration of phenylalanine increased linearly over $6 \mathrm{~h}, \mathrm{~d} C_{\text {Phe }} / \mathrm{d} t$ and $\mathrm{d} f_{\text {Phe }} / \mathrm{d} t$ were computed from the slopes of the time-series data. Then every 6-min estimates of $C_{\text {Phe }}$ and $f_{\text {Phe }}$ were derived based on $\mathrm{d} C_{\text {Phe }} / \mathrm{d} t$ and $\mathrm{d} f_{\text {Phe }} / \mathrm{d} t . f_{\text {Tyr }}$ was the average labelling percentage of tyrosine along the experiment. To determine the control $v_{c}$, emitted volatiles from day 2 control flowers fed with $10 \mathrm{mM}$ tyrosine were collected for 2,4 and $6 \mathrm{~h}$ starting at 18:00 h. No statistical differences were found in the scent profiles of flowers fed with $10 \mathrm{mM}$ tyrosine compared to control. Since the volatile amount was found to increase linearly (Supplementary Fig. $4 \mathrm{~b}$ ), $v_{\mathrm{c}}$ was assumed to be constant during the experimental period, and was derived from the slope of the time-series data. The $v_{c}$ for PhpCAT RNAi lines was subsequently determined by multiplying the control $v_{c}$ by the average fractional decrease of total emission observed in lines 9 and 17 (Fig. 5a). With these obtained values, $v_{1}$ and $v_{2}$ were calculated along the experimental period every $6 \mathrm{~min}$ using equations (3) and (4). The whole simulation was performed in Matlab R2013a environment (The MathWorks, Inc., Natick, MA). Variances in the estimated slopes were derived with a standard linear regression procedure as described ${ }^{60}$, while setting the intercepts as constants was based on experimental measurements. Since flux values are a function of the estimated trend slopes and other experimental measurements, flux variances can be derived by considering the propagation of errors based on the following equation:

$$
\sigma_{y}^{2}=\sum_{i=1}^{n} \sigma_{x_{i}}^{2}\left(\frac{\partial y}{\partial x_{i}}\right)^{2}
$$

where $y=f\left(x_{1}, \ldots, x_{\mathrm{n}}\right)$.

Preparation of plastids. Plastids were isolated from approximately $25 \mathrm{~g}$ of 1-3day-old petals of control, PhpCAT-RNAi line 9 and PhpCAT overexpression line 3 petunia plants. Flowers were kept in the dark for $2 \mathrm{~h}$ prior to plastid preparations to deplete starch. Petals were placed in a chilled blender containing $200 \mathrm{ml}$ ice-cold medium A (0.5 M sorbitol, $10 \mathrm{mM}$ MES/NaOH pH 6.3, $4 \mathrm{mM} \mathrm{Na-ascorbate,} 4 \mathrm{mM}$ cysteine, $2 \mathrm{mM} \mathrm{MgCl}_{2}, 1.5 \mathrm{mM} \mathrm{KH}_{2} \mathrm{PO}_{4}, 1 \mathrm{mM} \mathrm{MnCl}, 1 \mathrm{mM}$ EDTA-Na 2 ) and homogenized with three 2-s pulses. The resulting homogenate was filtered through two layers of Miracloth (Calbiochem, La Jolla, CA). An aliquot of filtrate (crude extract) was taken from each preparation and set aside, and the remainder was centrifuged at $6,000 \mathrm{~g}, 4^{\circ} \mathrm{C}$ for $5 \mathrm{~min}$. The pellet was washed twice in medium B (0.33 M sorbitol, $10 \mathrm{mM} \mathrm{MES} / \mathrm{NaOH} \mathrm{pH}$ 7.6), resuspended in $2 \mathrm{ml}$ of medium $\mathrm{B}$, and layered over a discontinuous Percoll ${ }^{\mathrm{TM}}$ gradient consisting of $80 \%(2 \mathrm{ml})$ and $25 \%(6 \mathrm{ml})$ Percoll ${ }^{\mathrm{TM}}$ prepared in medium B. After centrifugation $\left(9,200 \mathrm{~g}, 4^{\circ} \mathrm{C}\right.$ for $20 \mathrm{~min}$ in a swinging bucket rotor with no brake applied), the intact plastid fractions (approximately $1.5 \mathrm{ml}$ ) were collected from the interface of the Percoll ${ }^{\mathrm{TM}}$ layers, washed two times in medium $\mathrm{B}$, and finally resuspended in $50 \mathrm{mM}$ $\mathrm{NaH}_{2} \mathrm{PO}_{4}(\mathrm{pH} 8.0)$ with $5 \%(\mathrm{v} / \mathrm{v})$ glycerol, flash frozen and stored at $-80^{\circ} \mathrm{C}$.

Immunoblots. Immunoblot analysis was performed essentially as described previously ${ }^{61}$ using petunia crude extracts and plastids, E. coli crude extracts, and the purified PhpCAT ${ }_{53-583}$. Protein sample concentration was determined by the Bio-Rad Bradford protein assay. To solubilize membrane proteins, protein-loading dye containing $10 \%$ SDS was added to protein samples, which were then incubated at room temperature for $3 \mathrm{~h}$, and centrifuged at $5,000 \mathrm{~g}$ for $5 \mathrm{~min}$ to pellet debris. Immunodetection was performed on $2.5 \mu$ g protein using purified rabbit anti-PhpCAT polyclonal antibodies $(1: 3,000)$ generated against a synthetic peptide CMIDPDAPFSGAFMG (Genscript, Piscataway, NJ; http://www.genscript.com/ custom-polyclonal-antibody-production-services.html). A goat anti-rabbit IgG horseradish peroxidase conjugate $(1: 30,000)$ was used as secondary antibody (Bio-Rad, Hercules, CA; 170-6515). Antigen bands were visualized using an enhanced chemiluminescence reagent (PerkinElmer, Waltham, MA) according to the manufacturer's protocol, and exposing the gels on Eastman Kodak X-OMART 
AR film. An uncropped immunoblot corresponding to Fig. 4a is shown in Supplementary Fig. 9.

Chorismate mutase assays. CM assays were performed using protein prepared from petunia plastids according to a previously published method ${ }^{62}$. Briefly, corolla tissue was collected from day 2 flowers at 21:00 h, immediately frozen in liquid nitrogen, ground with mortar and pestle, and lyophilized at $-80^{\circ} \mathrm{C}, 0.01 \mathrm{mbar}$ for a minimum of $72 \mathrm{~h}$. Approximately $0.3 \mathrm{~g}$ of dried tissue was resuspended in $2.5 \mathrm{ml}$ heptane/tetrachloroethylene (density $=1.32 \mathrm{~g} \mathrm{ml}^{-1}$ ) and homogenized in a ball mill. The resulting suspension was layered atop a freshly prepared heptane/ tetrachloroethylene density gradient, then centrifuged for $90 \mathrm{~min}$ at $13,000 \mathrm{~g}, 4^{\circ} \mathrm{C}$. The resolved gradient was divided into six fractions; each fraction was divided into half and all solvent evaporated under nitrogen flow. For enzyme assays, the resulting residue was resuspended in $1 \mathrm{ml}$ of protein resuspension buffer $(50 \mathrm{mM}$ Tricine- $\mathrm{NaOH}, 10 \%$ glycerol, $\mathrm{pH}$ 8.4). Insoluble debris was pelleted by centrifugation, and the supernatant desalted into protein resuspension buffer before use in assays. Marker assays were completed for plastids (NADPglyceraldehyde-3-phosphate dehydrogenase) and cytosol (alcohol dehydrogenase) as described previously ${ }^{63,64}$. Plastid content peaked in the second heaviest fraction (7-12 $\mathrm{ml}$ as measured from the bottom of the gradient, approximate density $1.49-1.45 \mathrm{~g} \mathrm{ml}^{-1}$ ), as $46 \%$ of total recovered GAPDH activity was found in that fraction, compared to $18 \%$ of $\mathrm{ADH}$ activity. This fraction was used for subsequent analysis.

$\mathrm{CM}$ assays were performed as described previously ${ }^{10}$, except that they were adapted to high-performance liquid chromatography detection as follows. Following reaction incubation and subsequent conversion of prephenate to phenylpyruvate by acid hydrolysis, reactions were neutralized by addition of $50 \mu \mathrm{l}$ of $1 \mathrm{M} \mathrm{NaOH}$, and $10 \mu \mathrm{l}$ of the reaction mixture was analysed by high-performance liquid chromatography using the Waters Atlantis dC18 column $(3 \mu \mathrm{m}$,

$2.1 \times 150 \mathrm{~mm}$ ) held at $35^{\circ} \mathrm{C}$, with an 8 -min linear gradient of $5-70 \%$ acetonitrile in $1 \%$ formic acid at a flow rate of $0.4 \mathrm{ml} \mathrm{min}^{-1}$. Phenylpyruvate and chorismate were detected by absorbance at $288 \mathrm{~nm}$ and quantified by comparison to authentic standards. For phenylpyruvate product confirmation, MS/MS was completed using an Agilent 6460 Triple Quadrupole LC-MS. Data were collected for product ion scans on a precursor $m / z$ of 163.1 in negative mode using a collision energy of $20 \mathrm{~V}$, a fragmentor voltage of $70 \mathrm{~V}$ and dwell time of $50 \mathrm{~ms}$. The jet stream ESI interface had a gas temperature of $325^{\circ} \mathrm{C}$, gas flow rate of $81 \mathrm{~min}^{-1}$, nebulizer pressure of $35 \mathrm{psi}$, sheath gas temperature of $250^{\circ} \mathrm{C}$, sheath gas flow rate of $71 \mathrm{~min}^{-1}$, capillary voltage of $3,000 \mathrm{~V}$ and nozzle voltage of $500 \mathrm{~V}$.

Targeted metabolite profiling. Petunia volatiles (benzaldehyde, benzyl alcohol, phenylacetaldehyde, methylbenzoate, phenylethanol, benzylacetate, eugenol, isoeugenol, vanillin, benzylbenzoate and phenylethylbenzoate) were collected from detached flowers of control, PhpCAT-RNAi and PhpCAT overexpression lines (minimum of three flowers per biological replicate) from 18:00 to 22:00 h on day 2 postanthesis by a closed-loop stripping method and analysed by gas chromatography-MS as previously described ${ }^{4}$. Internal pools of the aromatic amino acids and organic acids (shikimate, prephenate and arogenate) from control and transgenic tissues collected (minimum of eight flowers per biological replicate) at 20:00 h on day 2 postanthesis were quantified by gas chromatography- or LC-MS as previously described ${ }^{10}$.

\section{References}

1. Maeda, H. \& Dudareva, N. The shikimate pathway and aromatic amino acid biosynthesis in plants. Annu. Rev. Plant Biol. 63, 73-105 (2012).

2. Ekholm, R. Biosynthesis of thyroid hormones. Int. Rev. Cytol. 120, 243-288 (1990).

3. Fernstrom, J. D. \& Fernstrom, M. H. Tyrosine, phenylalanine, and catecholamine synthesis and function in the brain. J. Nutr. 137, 1539S-1547S (2007).

4. Haslam, E. in Shikimic Acid: Metabolism and Metabolites (Wiley, 1993).

5. Razal, R. A., Ellis, S., Singh, S., Lewis, N. G. \& Towers, G. H. N. Nitrogen recycling in phenylpropanoid metabolism. Phytochemistry 41, 31-35 (1996).

6. Bonawitz, N. D. \& Chapple, C. The genetics of lignin biosynthesis: connecting genotype to phenotype. Annu. Rev. Genet. 44, 337-363 (2010).

7. Dudareva, N., Klempien, A., Muhlemann, J. K. \& Kaplan, I. Biosynthesis, function and metabolic engineering of plant volatile organic compounds. New Phytol. 198, 16-32 (2013).

8. Vlot, A. C., Dempsey, D. \& Klessig, D. F. Salicylic acid, a multifaceted hormone to combat disease. Annu. Rev. Phytopathol. 47, 177-206 (2009).

9. Vogt, T. Phenylpropanoid biosynthesis: invited review. Mol. Plant 3, 2-20 (2009).

10. Maeda, H. et al. RNAi suppression of arogenate dehydratasel reveals that phenylalanine is synthesized predominantly via the arogenate pathway in petunia petals. Plant Cell 22, 832-849 (2010).

11. Maeda, H., Yoo, H. \& Dudareva, N. Prephenate aminotransferase directs plant phenylalanine biosynthesis via arogenate. Nat. Chem. Biol. 7, 19-21 (2011).
12. Yoo, H. et al. An alternative pathway contributes to phenylalanine biosynthesis in plants via a cytosolic tyrosine:phenylpyruvate aminotransferase. Nat. Commun. 4, 2833 (2013).

13. Carruthers, A. \& Melchior, D. L. Study of the relationship between bilayer water permeability and bilayer physical state. Biochemistry 22, 5797-5807 (1983).

14. Chakrabarti, A. C. \& Deamer, D. W. Permeability of lipid bilayers to amino acids and phosphate. Biochim. Biophys. Acta 1111, 171-177 (1992).

15. Weber, A. P. M., Schwacke, R. \& Flügge, U. Solute transporters of the plastid envelope membrane. Annu. Rev. Plant Biol. 56, 133-164 (2005).

16. Pohlmeyer, K., Soll, J., Steinkamp, T., Hinnah, S. \& Wagner, R. Isolation and characterization of an amino acid-selective channel protein present in the chloroplastic outer envelope membrane. Proc. Natl Acad. Sci. USA 94, 9504-9509 (1997).

17. Pudelski, B., Kraus, S., Soll, J. \& Philippar, K. The plant PRAT proteins preprotein and amino acid transport in mitochondria and chloroplasts. Plant Biol. 12, 42-55 (2010).

18. Pudelski, B. et al. The plastid outer envelope protein OEP16 affects metabolic fluxes during ABA-controlled seed development and germination. J. Exp. Bot. 63, 1919-1936 (2012).

19. Weber, A. P. M. \& Linka, N. Connecting the plastid: transporters of the plastid envelope and their role in linking plastidial with cytosolic metabolism. Annu. Rev. Plant Biol. 62, 53-77 (2011).

20. Reyes-Prieto, A. \& Moustafa, A. Plastid-localized amino acid biosynthetic pathways of Plantae are predominantly composed of non-cyanobacterial enzymes. Sci. Rep. 2, 955 (2012).

21. Yang, H., Bogner, M., Stierhof, Y. \& Ludewig, U. $\mathrm{H}^{+}$-independent glutamine transport in plant root tips. PLoS ONE 5, e8917 (2010).

22. Yang, H., Krebs, M., Stierhof, Y. \& Ludewig, U. Characterization of the putative amino acid transporter genes AtCAT2, 3 \& 4: the tonoplast localized AtCAT2 regulates soluble leaf amino acids. J. Plant Physiol. 171, 594-601 (2014).

23. Su, Y., Frommer, W. B. \& Ludewig, U. Molecular and functional characterization of a family of amino acid transporters from Arabidopsis. Plant Physiol. 136, 3104-3113 (2004).

24. Tegeder, M. \& Rentsch, D. Uptake and partitioning of amino acids and peptides. Mol. Plant 3, 997-1011 (2010).

25. Ljungdahl, P. O. \& Daignan-Fornier, B. Regulation of amino acid, nucleotide, and phosphate metabolism in Saccharomyces cerevisiae. Genetics 190, 885-929 (2012).

26. Pi, J., Wookey, P. J. \& Pittard, A. J. Cloning and sequencing of the pheP gene, which encodes the phenylalanine-specific transport system of Escherichia coli. J. Bacteriol. 173, 3622-3629 (1991).

27. Chye, M. L., Guest, J. R. \& Pittard, J. Cloning of the aroP gene and identification of its product in Escherichia coli K-12. J. Bacteriol. 167, 749-753 (1986).

28. Brown, K. D. Formation of aromatic amino acid pools in Escherichia coli K-12. J. Bacteriol 104, 177-188 (1970).

29. Koyanagi, T., Katayama, T., Suzuki, H. \& Kumagai, H. Identification of the LIV-I/LS system as the third phenylalanine transporter in Escherichia coli K-12. J. Bacteriol. 186, 343-350 (2004).

30. Boatright, J. et al. Understanding in vivo benzenoid metabolism in petunia petal tissue. Plant Physiol. 135, 1993-2011 (2004).

31. Verdonk, J. C. et al. Regulation of floral scent production in petunia revealed by targeted metabolomics. Phytochemistry 62, 997-1008 (2003).

32. Bordych, C., Eisenhut, M., Pick, T. R., Kuelahoglu, C. \& Weber, A. P. M. Co-expression analysis as tool for the discovery of transport proteins in photorespiration. Plant Biol. 15, 686-693 (2013).

33. Pick, T. R. et al. PLGG1, a plastidic glycolate glycerate transporter, is required for photorespiration and defines a unique class of metabolite transporters. Proc. Natl Acad. Sci. USA 110, 3185-3190 (2013).

34. Qualley, A. V., Widhalm, J. R., Adebesin, F., Kish, C. M. \& Dudareva, N. Completion of the core $\beta$-oxidative pathway of benzoic acid biosynthesis in plants. Proc. Natl Acad. Sci. USA 109, 16383-16388 (2012).

35. Klempien, A. et al. Contribution of CoA ligases to benzenoid biosynthesis in petunia flowers. Plant Cell 24, 2015-2030 (2012).

36. Colquhoun, T. A. et al. A petunia chorismate mutase specialized for the production of floral volatiles. Plant J. 61, 145-155 (2010).

37. Van Moerkercke, A., Schauvinhold, I., Pichersky, E., Haring, M. A. \& Schuurink, R. C. A plant thiolase involved in benzoic acid biosynthesis and volatile benzenoid production. Plant J. 60, 292-302 (2009).

38. Hammes, U. Z., Nielsen, E., Honaas, L. A., Taylor, C. G. \& Schachtman, D. P. AtCAT6, a sink-tissue-localized transporter for essential amino acids in Arabidopsis. Plant J. 48, 414-426 (2006).

39. Kolosova, N., Gorenstein, N., Kish, C. M. \& Dudareva, N. Regulation of circadian methyl benzoate emission in diurnally and nocturnally emitting plants. Plant Cell 13, 2333-2347 (2001).

40. Tjaden, J., Schwöppe, C., Möhlmann, T., Quick, P. W. \& Neuhaus, H. E. Expression of a plastidic ATP/ADP transporter gene in Escherichia coli leads to 
a functional adenine nucleotide transport system in the bacterial cytoplasmic membrane. J. Biol. Chem. 273, 9630-9636 (1998).

41. Klaus, S. M. J. et al. Higher plant plastids and cyanobacteria have folate carriers related to those of trypanosomatids. J. Biol. Chem. 280, 38457-38463 (2005).

42. Bedhomme, M. et al. Folate metabolism in plants: an Arabidopsis homolog of the mammalian mitochondrial folate transporter mediates folate import into chloroplasts. J. Biol. Chem. 280, 34823-34831 (2005).

43. Pavón, L. R. et al. Arabidopsis ANTR1 is a thylakoid $\mathrm{Na}+$-dependent phosphate transporter: functional characterization in Escherichia coli. J. Biol. Chem. 283, 13520-13527 (2008).

44. Eudes, A. et al. Identification of transport-critical residues in a folate transporter from the folate-biopterin transporter (FBT) family. J. Biol. Chem. 285, 2867-2875 (2010).

45. Furumoto, T. et al. A plastidial sodium-dependent pyruvate transporter. Nature 476, 472-475 (2011).

46. Orlova, I. et al. Reduction of benzenoid synthesis in petunia flowers reveals multiple pathways to benzoic acid and enhancement in auxin transport. Plant Cell 18, 3458-3475 (2006).

47. Eberhard, J. et al. Cytosolic and plastidic chorismate mutase isozymes from Arabidopsis thaliana: molecular characterization and enzymatic properties. Plant J. 10, 815-821 (1996).

48. Martinoia, E., Meyer, S., De Angeli, A. \& Nagy, R. Vacuolar transporters in their physiological context. Annu. Rev. Plant Biol. 63, 183-213 (2012).

49. Tohge, T. et al. Toward the storage metabolome: profiling the barley vacuole. Plant Physiol. 157, 1469-1482 (2011).

50. Benkeblia, N., Shinano, T. \& Osaki, M. Metabolite profiling and assessment of metabolome compartmentation of soybean leaves using non-aqueous fractionation and GC-MS analysis. Metabolomics 3, 297-305 (2007).

51. Heinig, U., Gutensohn, M., Dudareva, N. \& Aharoni, A. The challenges of cellular compartmentalization in plant metabolic engineering. Curr. Opin. Biotechnol. 24, 239-246 (2013).

52. Gleave, A. A versatile binary vector system with a T-DNA organisational structure conducive to efficient integration of cloned DNA into the plant genome. Plant Mol. Biol. 20, 1203-1207 (1992).

53. Horsch, R. B. et al. A simple and general method for transferring genes into plants. Science 227, 1229-1231 (1985).

54. Grabherr, M. G. et al. Full-length transcriptome assembly from RNA-Seq data without a reference genome. Nat. Biotechnol. 29, 644-652 (2011).

55. Götz, S. et al. High-throughput functional annotation and data mining with the Blast2GO suite. Nucleic Acids Res. 36, 3420-3435 (2008)

56. Robinson, M. D., McCarthy, D. J. \& Smyth, G. K. edgeR: a Bioconductor package for differential expression analysis of digital gene expression data. Bioinformatics 26, 139-140 (2010).

57. Smyth, G. K. in Bioinformatics and Computational Biology Solutions Using $R$ and Bioconductor: Limma: Linear Models for Microarray Data. (eds Gentleman, R., Carey, V., Dudoit, S., Irizarry, R. \& Huber, W.) 397-420 (Springer, 2005).

58. Trapnell, C. et al. Transcript assembly and quantification by RNA-Seq reveals unannotated transcripts and isoform switching during cell differentiation. Nat. Biotechnol. 28, 511-515 (2010)
59. Karimi, M., Inze, D. \& Depicker, A. GATEWAY vectors for Agrobacteriummediated plant transformation. Trends Plant Sci. 7, 193-195 (2002).

60. Press, W. H. in Numberical Recipes. 3rd edn: The Art of Scientific Computing (Cambridge University Press, 2007)

61. Dudareva, N. et al. Developmental regulation of methyl benzoate biosynthesis and emission in snapdragon flowers. Plant Cell. 12, 949-961 (2000).

62. Krueger, S. et al. A topological map of the compartmentalized Arabidopsis thaliana leaf metabolome. PLoS ONE 6, e17806 (2011).

63. Smith, A. M. \& Rees, T. Pathways of carbohydrate fermentation in the roots of marsh plants. Planta 146, 327-334 (1979).

64. van Oostende, C., Widhalm, J. R. \& Basset, G. J. C. Detection and quantification of vitamin $K_{1}$ quinol in leaf tissues. Phytochemistry 69, 2457-2462 (2008).

\section{Acknowledgements}

We thank the Scott Briggs laboratory for assistance with the immunoblots. This work was supported by grant DE-SC0008628 from the US Department of Energy to N.D. and J.A.M. and by the Gordon and Betty Moore Foundation through grant GBMF2550.02 to the Life Sciences Research Foundation to J.R.W.

\section{Author contributions}

J.R.W., M.G. and N.D. conceived the project and designed research; J.R.W., M.G., H.Y. Y.Q., F.A., L.G., R.J., J.H.L. and R.M.M. performed research; J.R.W., M.G., L.G., J.A.M., D.R. and N.D. analysed the data; J.T.S. and J.T. analysed RNA-Seq data; J.R.W. and N.D. wrote the paper. All authors read and edited the manuscript.

\section{Additional information}

Accession codes: The GenBank accession number for the PhpCAT sequence mentioned in this article is KP694324. The petunia RNA-seq data set has been deposited in NCBI Gene Expression Omnibus (GEO) under accession code GSE70948.

Supplementary Information accompanies this paper at http://www.nature.com/ naturecommunications

Competing financial interests: The authors declare no competing financial interests.

Reprints and permission information is available online at http://npg.nature.com/ reprintsandpermissions/

How to cite this article: Widhalm, J. R. et al. Identification of a plastidial phenylalanine exporter that influences flux distribution through the phenylalanine biosynthetic network. Nat. Commun. 6:8142 doi: 10.1038/ncomms9142 (2015).

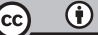

This work is licensed under a Creative Commons Attribution 4.0 International License. The images or other third party material in this article are included in the article's Creative Commons license, unless indicated otherwise in the credit line; if the material is not included under the Creative Commons license, users will need to obtain permission from the license holder to reproduce the material To view a copy of this license, visit http://creativecommons.org/licenses/by/4.0/ 